\title{
Experimental and theoretical cross sections for molecular-frame electron-impact excitation-ionization of $\mathrm{D}_{2}$
}

\author{
Julian C. A. Lower, ${ }^{1}$ Esam Ali, ${ }^{2}$ Susan Bellm, ${ }^{3}$ Erich Weigold, ${ }^{3}$ Allison Harris, ${ }^{4}$ C. G. Ning, ${ }^{5}$ and Don Madison ${ }^{2}$ \\ ${ }^{1}$ Institut für Kernphysik, Max-von-Laue-Strasse 1, 60438 Frankfurt am Main, Germany \\ ${ }^{2}$ Missouri University of Science and Technology, Rolla, Missouri 65409, USA \\ ${ }^{3}$ AMPL, RSPE, The Australian National University, Canberra, Australian Capital Territory 0200, Australia \\ ${ }^{4}$ Illinois State University, Normal, Illinois 61790, USA \\ ${ }^{5}$ Department of Physics and State Key Laboratory of Low-Dimensional Quantum Physics, Tsinghua University, Beijing 100084, China
}

(Received 12 August 2013; published 5 December 2013)

\begin{abstract}
We present both experimental and theoretical results for the dissociative ionization of $\mathrm{D}_{2}$ molecules induced by electron impact. Cross sections are determined in the molecular frame and are fully differential in the energies and emission angles of the dissociation fragments. Transitions are considered from the $X^{1} \Sigma_{g}{ }^{+}$electronic ground state of $\mathrm{D}_{2}$ to the $2 s \sigma_{g}, 2 p \pi_{u}$ and $2 p \sigma_{u}$ excited states of $\mathrm{D}_{2}{ }^{+}$. The experimental results are compared to calculations performed within the molecular four-body distorted-wave framework to describe the multicenter nature of the scattering process. The cross sections reveal a dramatic dependence on both the alignment of the internuclear axis with respect to the direction of the projectile momentum and on the symmetry of the excited dissociating state which is energetically resolved.
\end{abstract}

DOI: 10.1103/PhysRevA.88.062705

PACS number(s): $34.80 . \mathrm{Dp}, 34.80 . \mathrm{Nz}$

\section{INTRODUCTION}

The electron-impact-induced ionization and fragmentation of molecules is a ubiquitous process of biological, industrial, and theoretical relevance. It plays a central role in the physics and chemistry of the upper atmosphere, the operation of discharges and lasers, radiation-induced damage in biological material, and plasma etching processes [1-3]. It is a process which describes both the removal of a parent molecule from a chemical environment and the liberation of atoms and molecules in neutral and charged states. The fragments themselves are often highly reactive due to unpaired electrons or their charge state and drive additional reactions in their local environment.

From a technological perspective, the extent to which such processes can be controlled is limited by our understanding of the physical mechanisms which underpin them and our ability to predict reaction rates under disparate physical conditions. Of great assistance to achieving these goals are measurements in which fragments are measured in time coincidence and in which the reaction kinematics are fully determined. Such measurements provide highly differential cross sections which describe how the reaction probabilities for particular reaction pathways depend on the momenta of the projectile electron and the scattered electrons and on the momenta and internal energy states of the parent molecule and its charged and neutral fragmentation products. Crucial to the interpretation of such results is careful comparison with calculations. The generation of fully differential cross sections for the molecularfragmentation process considered here is extremely challenging due to complexities in describing electron scattering from a many-centered scattering potential and modeling the many-body dynamics which is mediated through the Coulomb potential. Comparison of theory with experimental data can be used to hone theory, establishing the relative merits and ranges of validity for the various approximations presently required to render calculations tractable. If a sound theoretical framework can be established to describe the problem, it can then be used to predict reaction rates and pathways in kinematical regions uncovered by or inaccessible to experimental investigation.

Previous studies of alignment-resolved $(e, 2 e)$ studies of $\mathrm{H}_{2}$ were extensively reviewed in [4] so only a brief account will be given here. Pioneering experiments were undertaken by the Sendai group [5-8] who, using hemispherical electrostatic energy analyzers, performed measurements under conditions of high-momentum transfer. Experimental and theoretical results were presented at impact energies of 1.2 and $2.0 \mathrm{keV}$ to the $2 s \sigma_{g}$ and $2 p \sigma_{u}$ states and for molecular alignments where the internuclear axis was directed orthogonally to the projectile-electron direction. A strong alignment dependence in the measured $(e, 2 e)$ count rate was observed with indications that for transitions to the $2 p \sigma_{u}$ state, the two outgoing electrons escape preferentially so as to leave the ion recoil momenta along the molecular axis.

In contrast, measurements at lower impact energies were performed by the Heidelberg group [9-12]. Using a reaction microscope their measurements were performed at an impact energy of $200 \mathrm{eV}$ under conditions of highly asymmetric energy sharing between the two $(e, 2 e)$ electrons $[9,10]$ and at lower impact energies of $31.5 \mathrm{eV}$ [12] and $54 \mathrm{eV}$ [11] in later publications. All of these studies focused on transitions from the $X^{1} \Sigma_{g}{ }^{+}$electronic ground state of $\mathrm{H}_{2}$ to the vibrational continuum of the $1 s \sigma_{g}$ ground state of $\mathrm{H}_{2}{ }^{+}$. In $[9,10]$ the experimental results were compared to molecular three-body distorted-wave (M3DW) calculations [13-15] and another calculation involving atomic cross sections multiplied by an alignment-dependent interference factor [16]. In [12] the effects of projectile-nucleus scattering were explored through measurements in noncoplanar scattering geometry under which conditions these effects are enhanced [17] and the results were compared to those for electron-helium scattering under similar kinematics. Comparison of the experimental results was made to time-dependent close-coupling (TDCC) [18-21] and convergent close-coupling (CCC) $[22,23]$ calculations in addition to M3DW results. 
Other low-energy measurements were reported by the Canberra group [24]. At an impact energy of $178 \mathrm{eV}$ they investigated transitions to the $2 s \sigma_{g}$ and $2 p \pi_{u}$ excited states of $\mathrm{H}_{2}{ }^{+}$. In contrast to the pioneering work of the Sendai group $[5,6,8]$ they were able to study all molecular alignments, not just "side-on collisions" of the primary electron with the target molecule. However, due to limitations in their ion-energy measurement range, they were unable to access transitions to the $2 p \sigma_{u}$ state of $\mathrm{H}_{2}{ }^{+}$as achieved by the Sendai group.

Here we present experimental and theoretical results for the the dissociative ionization of $\mathrm{D}_{2}$ at an electron-impact energy of $178 \mathrm{eV}$. From the perspective of its electronic structure and geometry, the $\mathrm{D}_{2}\left(\mathrm{H}_{2}\right)$ molecule presents the simplest neutral molecular target to explore mechanisms of dissociative ionization. The present experimental results extend earlier results [24] for $\mathrm{H}_{2}$ which were measured under identical reaction kinematics but restricted to transitions to the $2 s \sigma_{g}$ and $2 p \pi_{u}$ excited states of the $\mathrm{H}_{2}{ }^{+}$ion. While the electronic structure of $\mathrm{D}_{2}$ is essentially the same as that of $\mathrm{H}_{2}$, its greater nuclear masses leads to substantially lower fragment velocities associated with dissociative transitions. These lower fragment velocities, in conjunction with improvements made to our ion spectrometer, enabled us to increase the amount of ionmomentum phase space over which we could simultaneously measure compared to our previous work. As a consequence we are able to measure transitions to the $2 p \sigma_{u}$ in addition to those to the $2 s \sigma_{g}$ and $2 p \pi_{u}$ excited states of the $\mathrm{D}_{2}{ }^{+}$ ion. By measuring deuterons and electrons in a coincidence experiment we are able to determine not only the dependence of the dissociative-ionization process on the alignment of the internuclear axis with respect to the momenta of the projectile and scattered electrons, but also its dependence on the symmetry of the $\mathrm{D}_{2}{ }^{+}$electronic state excited in the process.

\section{REACTION GEOMETRY AND REACTION PATHWAYS}

A schematic representation of the dissociative-ionization process under consideration and the adopted reaction kinematics is shown schematically in Fig. 1. Mathematically, it can be described by the equation

$$
\begin{aligned}
e_{i}^{-}\left(\mathbf{p}_{\mathbf{i}}\right)+\mathrm{D}_{2}\left(\theta_{\mathrm{D}}, \phi_{\mathrm{D}}\right) \rightarrow & \mathrm{D}\left(n, \mathbf{p}_{\mathbf{D}}\right)+\mathrm{D}^{+}\left(\mathbf{p}_{\mathbf{D}^{+}}\right) \\
& +e_{a}^{-}\left(\mathbf{p}_{\mathbf{a}}\right)+e_{b}^{-}\left(\mathbf{p}_{\mathbf{b}}\right) .
\end{aligned}
$$

Here $e_{i}^{-}\left(\mathbf{p}_{\mathbf{i}}\right), e_{a}^{-}\left(\mathbf{p}_{\mathbf{a}}\right)$, and $e_{b}^{-}\left(\mathbf{p}_{\mathbf{b}}\right)$ represent incident, scattered, and ejected electrons of respective momenta $\mathbf{p}_{\mathbf{i}}, \mathbf{p}_{\mathbf{a}}$, and $\mathbf{p}_{\mathbf{b}}$ (energies $E_{i}, E_{a}$, and $E_{b}$ ). The momentum transfer from the projectile to the target is characterized by the momentumtransfer vector $\boldsymbol{K}$, defined through the expression $\boldsymbol{K}=\mathbf{p}_{\mathbf{i}}-$ $\mathbf{p}_{\mathbf{a}}$, where $\mathbf{p}_{\mathbf{a}}$ is the faster of the two final-state electrons. Due to the imaging properties of our electron-energy analyzers, only collision events for which the momenta of the incident electron and the two final-state continuum electrons are constrained to a common plane, the so-called scattering plane, are measured in the present experiment. $\mathrm{D}_{2}\left(\theta_{\mathrm{D}}, \phi_{\mathrm{D}}\right)$ represents the parent deuterium molecule at rest in the laboratory frame and in the electronic ground state. Its alignment angle with respect to the scattering plane and the projectile momentum vector is described by the polar coordinates $\left(\theta_{\mathrm{D}}, \phi_{\mathrm{D}}\right)$. $\mathrm{D}\left(n, \mathbf{p}_{\mathbf{D}}\right)$ represents the fragment deuterium atom of momentum $\mathbf{p}_{\mathbf{D}}$ and principle (a) Before Collision

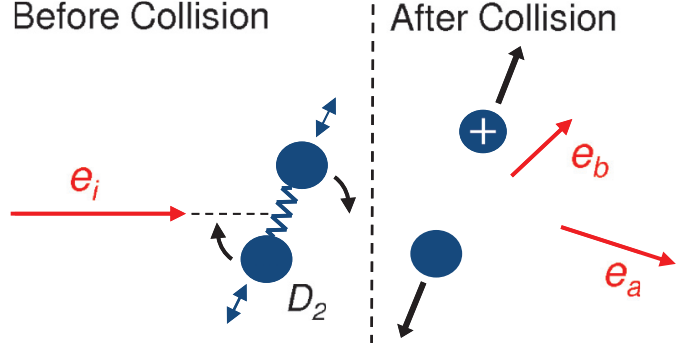

(b)

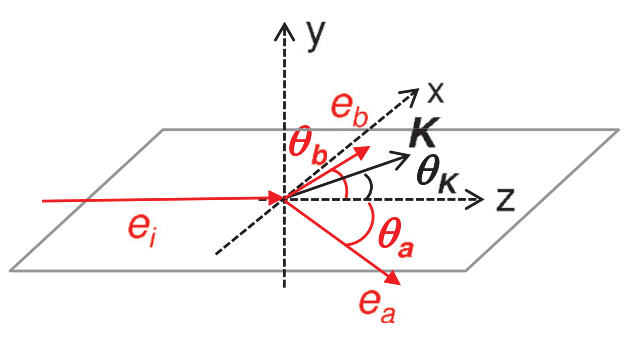

(c)

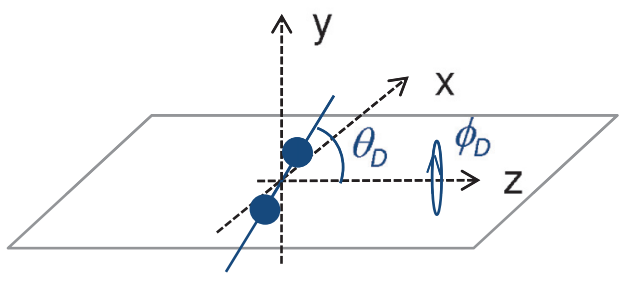

FIG. 1. (Color online) Panel (a) Schematic representation of dissociative-ionization process considered in this paper. A projectile electron $e_{i}$ collides with a deuterium molecule, liberating two scattered electrons $e_{a}$ and $e_{b}$, a deuteron and a deuterium atom in its ground state or in one of its excited states. Panel (b) Reaction kinematics. The two scattered electrons are detected in a plane containing the incident electron beam and are detected at angles $\theta_{a}$ and $\theta_{b}$ on opposite sides of the beam. The direction of momentum transfer $\theta_{K}$ varies with the scattering angle $\theta_{a}$. Panel (c) The alignment of the molecular axis, at the instant of ionization, is defined through the angles $\left(\theta_{\mathrm{D}}, \phi_{\mathrm{D}}\right)$ and is inferred from the asymptotic trajectory of the deuteron fragment. The dissociation rate depends on the molecular alignment, the momenta of the projectile and scattered electrons, and on the molecular and ionic states involved in the dissociative transition.

quantum number $n$ and $\mathrm{D}^{+}\left(\mathbf{p}_{\mathbf{D}^{+}}\right)$represents a deuteron of momentum $\mathbf{p}_{\mathbf{D}^{+}}$(energy $E_{\mathrm{D}^{+}}$).

Deuterons emitted over the full $4 \pi$ solid angle are detected in the present measurement. Both the fragmentation rate and the $n$-state distribution of the deuterium fragment atoms are shown to depend strongly on the alignment angle $\left(\theta_{\mathrm{D}}, \phi_{\mathrm{D}}\right)$. For the low impact energy and low values of momentum transfer $\boldsymbol{K}$ considered, momentum transfer from the incident electron to the $\mathrm{D}_{2}$ center-of-mass and momentum transfer between the scattered electrons and the fragment nuclei is negligible. Thus, on dissociation of the excited $\mathrm{D}_{2}{ }^{+}, \mathbf{p}_{\mathbf{D}^{+}} \cong-\mathbf{p}_{\mathbf{D}}$ and for a known value of $\mathbf{p}_{\mathbf{i}}$, determination of $\mathbf{p}_{\mathbf{a}}, \mathbf{p}_{\mathbf{b}}$, and $\mathbf{p}_{\mathbf{D}^{+}}$ completely determines the reaction kinematics. Furthermore, by invoking energy conservation, the appearance energy $A(n)$ for transitions to the quantum state $n$ of the residual deuterium atom is determined through the relation

$$
A(n)=\varepsilon_{b}-2 E_{\mathrm{D}^{+}} .
$$




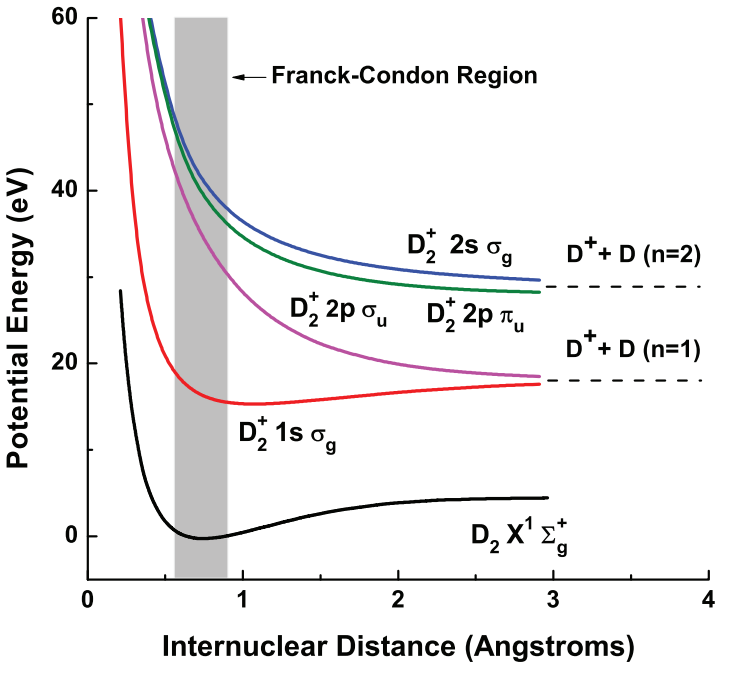

FIG. 2. (Color online) Potential energy of $\mathrm{D}_{2}$ and $\mathrm{D}_{2}{ }^{+}$(approximated by fitting hydrogen data from [26]) as a function of internuclear distance, for the states relevant to the present study. The measurement energetically resolves transitions to the $2 s \sigma_{g}$ and $2 p \pi_{u}$ states from those to the $1 s \sigma_{g}$ and $2 p \sigma_{u}$ states due to the $10.2-\mathrm{eV}$ separation between their respective dissociation limits (indicated by the dashed lines). Transitions to the $1 s \sigma_{g}$ and $2 p \sigma_{u}$ states can be easily resolved from one another due to their well-separated deuteron energy distributions.

Here $\varepsilon_{b}$ is the electron binding energy, defined by the expression $\varepsilon_{b}=E_{i}-E_{a}-E_{b}$, and $2 E_{\mathrm{D}^{+}}$accounts for the kinetic energy shared between the deuteron and the deuterium atom. This expression allows ionization events to be sorted according to the dissociation limits of the respective transitions with which they are associated. Finally, determination of $\mathbf{p}_{\mathbf{D}^{+}}$ enables the molecular alignment at the time of ionization to be inferred and alignment-resolved data to be obtained [25].

Figure 2 shows a simplified potential energy diagram for the deuterium molecule and molecular ion. Only the four states of $\mathrm{D}_{2}{ }^{+}$are shown which, under the adopted reaction kinematics and for the processes presently under investigation, are involved in the the strongest transitions. Dissociative ionization of the $\mathrm{D}_{2}$ molecule may occur through a number of distinct pathways. First, it can proceed directly via transitions from the $X^{1} \Sigma_{g}{ }^{+}$electronic ground state of $\mathrm{D}_{2}$ to the vibrational continuum of the $1 s \sigma_{g}$ ground state of $\mathrm{D}_{2}{ }^{+}$. Deuterons and deuterium atoms produced through these transition are released with low values of kinetic energy (typically $\ll 1 \mathrm{eV}$ ). This ground-state dissociation process (termed GSD in [9]) has been extensively studied [5-11] in recent years. Second, dissociation may occur by the direct excitation of both target electrons. In this double-electron-excitation (DEE) process, one electron is excited to the ionization continuum and the other to an excited state of $\mathrm{D}_{2}{ }^{+}$(all excited states of $\mathrm{D}_{2}{ }^{+}$ are dissociative), leading to deuterons and deuterium atoms of higher values of kinetic energy (typically $2-10 \mathrm{eV}$ ). This ionization-excitation process is the dominant dissociativeionization mechanism under the present kinematics and is the focus of this study. In contrast to the first pathway involving the electronic excitation of only a single electron, this DEE process presents a considerably greater challenge to theory since it must be treated as a four-body problem instead of an effective three-body problem. Third, dissociative ionization may also occur indirectly through transitions to intermediate autoionizing states of $\mathrm{D}_{2}[9,10]$ (termed $\mathrm{AI}$ in [9]). However, in the present measurement only emitted-electron energies above $30 \mathrm{eV}$ are considered, thereby avoiding contributions from this resonance pathway. This restriction serves to simplify the data analysis by restricting the number of participating reaction pathways.

\section{EXPERIMENT}

Details of the measurement procedure have been described previously [24,27] so only a short summary is given here. A schematic representation of the experimental arrangement is presented in Fig. 2 of [24]. Briefly, an electron beam is generated through photoionization of a strained GaAs crystal photocathode under illumination by laser light. The beam is accelerated to $178 \mathrm{eV}$ and focused onto an effusive jet of $\mathrm{D}_{2}$ molecules, crossing the jet orthogonally to form a localized interaction region (around $1 \mathrm{~mm}$ extent in all three spatial directions). Electrons emitted within a plane containing the primary-electron beam are collected in one of two toroidal-sector electrostatic electron analyzers (see [28] for details). Each analyzer employs a position-and-timesensitive delay-line detector [29], enabling electron momenta to be reconstructed from the spatial and temporal electronarrival coordinates. One analyzer is adjusted to transmit electrons in the energy range $\Delta E_{a}$, where $90 \mathrm{eV} \leqslant E_{a} \leqslant$ $110 \mathrm{eV}$ over the angular range $10^{\circ} \leqslant \theta_{a} \leqslant 50^{\circ}$ on one side of the electron beam. The second analyzer measures electrons in the energy range $\Delta E_{b}$, where $30 \mathrm{eV} \leqslant E_{b} \leqslant 50 \mathrm{eV}$ over the angular range $40^{\circ} \leqslant \theta_{b} \leqslant 80^{\circ}$ on the other side of the electron beam (see Fig. 1). We note here that the projectile-electron energy $E_{i}$ and the average energies $\bar{E}_{a}$ and $\bar{E}_{b}$ for the two emitted electrons correspond to de Broglie wavelengths of $0.92,1.2$, and $1.9 \AA$, respectively; in contrast, the equilibrium internuclear separation for $\mathrm{D}_{2}$ is around $0.58 \AA$. Deuterons emitted over a $4 \pi$ solid angle were focused by a pulsed-field ion spectrometer onto a third delay-line detector. A schematic of the spectrometer showing simulated ion trajectories for $10 \mathrm{eV} \mathrm{N}^{+}$ions produced by SIMION [31] software is shown in Fig. 3. From measurement of the deuteron arrival positions and arrival times, their momenta were uniquely determined and the molecular alignment, at the instant of ionization, was inferred. The combined momentum coordinates of electrons and ions enabled partial cross sections describing transitions to the unresolved $2 s \sigma_{g}$ and $2 p \pi_{u}$ and to the $2 p \sigma_{u}$ excited states of $\mathrm{D}_{2}{ }^{+}$to be determined.

Crucial to the success of the present measurement was the application of time-dependent fields to identify, in time coincidence, two electrons and a deuteron derived from individual dissociative-ionization events and to suppress background signal resulting from dissociative-ionization events for which the associated $(e, 2 e)$ pair remained undetected (the electron analyzers, although highly efficient, measure $<0.1 \%$ of all ionization events). The timing scheme we employed is shown schematically in Fig. 4.

Briefly, the primary electron beam was pulsed with a $30 \%$ duty cycle at a frequency of $125 \mathrm{kHz}$ with a temporal pulse width of $2.5 \mu \mathrm{s}$. Between each electron pulse a "cleaning 


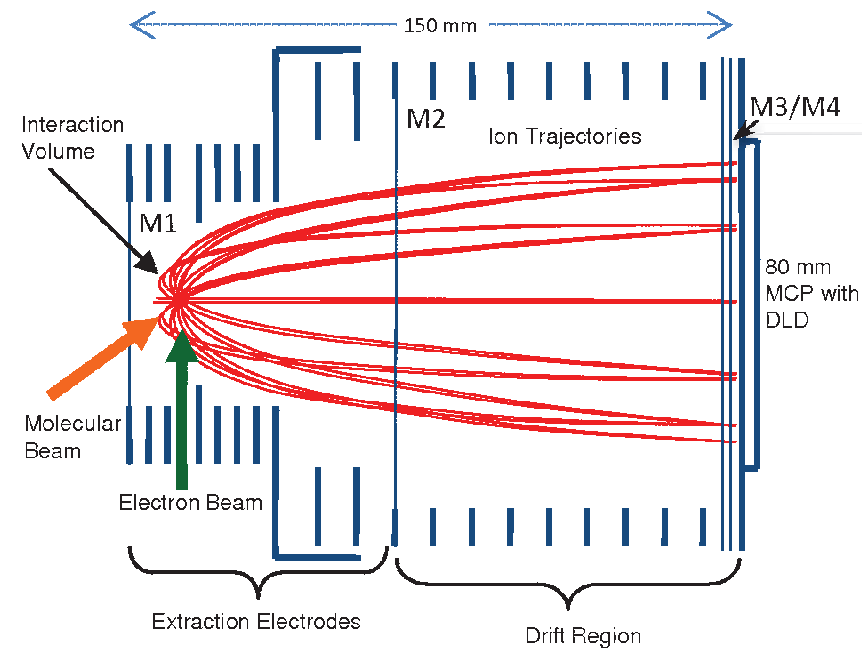

FIG. 3. (Color online) Cross-sectional view of the ion spectrometer. The molecular beam is admitted through a $0.8-\mathrm{mm}$ internal-bore needle (not shown) and crosses the electron beam at an angle of $90^{\circ}$. Ions are extracted by pulsing the potential of the mesh M1 which is coupled to the extraction electrodes and the grounded mesh M2 through a voltage-dividing resistor chain. The electrodes between meshes M2 and M3 and the meshes M3 and M4 themselves are maintained at a constant potential. The combination of separate ion acceleration and drift regions and the action of the lensing surface, arising from the potential difference across M2, creates conditions for spatial and temporal time focusing [30]. Ions are detected on an 80-mm-diameter microchannel-plate detector (MCP) equipped with a delay-line detector (DLD). Trajectories, simulated by SIMION software [31], are shown for $10-\mathrm{eV} \mathrm{N}^{+}$ions for emission angles in $30^{\circ}$ angular steps. See text for details.

cycle" was implemented to sweep away residual deuterons from the interaction region by applying a fast-rise-time $252-\mathrm{V}$ positive potential $V_{n}$ to the gas needle through which the molecular beam is introduced. If, at a time $t_{(e, 2 e)}$, an $(e, 2 e)$ ionization event was identified by the time-correlated arrival of two electrons at the electron detectors, an extraction field was generated within the ion spectrometer to collect deuteron fragments (see Fig. 3). This was achieved by applying a potential of $300 \mathrm{~V}$ to mesh M1 which is electrically bridged to the first ten extraction electrodes and the grounded mesh M2 through a resistor chain. Due to the finite flight times for electrons traversing the electron analyzers and the finite response time of our pulse-processing electronics, the time delay between the instant of ionization at time $t_{i}$ and the time at which the extraction voltages were applied $t_{\text {ext }}$ was $\sim 120 \mathrm{~ns}$, i.e., $t_{i}<t_{(e, 2 e)}<t_{\mathrm{ext}}$ with $t_{\mathrm{ext}}-t_{i} \sim 120 \mathrm{~ns}$. The extraction field was maintained for a period of $8 \mu \mathrm{s}$, a sufficient time for the associated deuteron fragment of up to $12 \mathrm{eV}$ kinetic energy to reach the ion detector. At the same time the needle potential was raised to the potential $V_{n}$, the value of $252 \mathrm{~V}$ chosen to optimize deuteron focusing onto the ion detector. Furthermore, upon detection of an $(e, 2 e)$ event and to reduce deuteron background, the electron-beam pulsing sequence was interrupted during the period of ion extraction by prematurely turning off the electron beam. This was achieved through the operation of a fast optical shutter positioned between the laser source and the photocathode.

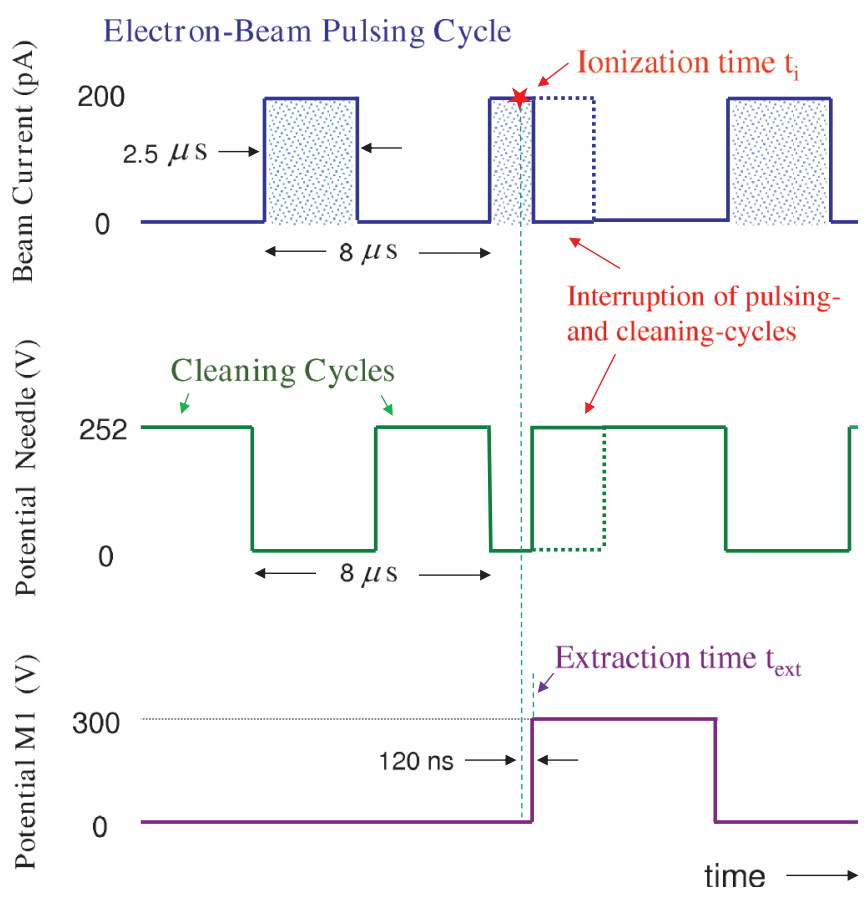

FIG. 4. (Color online) Pulsing scheme for the electron beam, for the potential applied to target-gas needle and for the potential applied to the mesh $\mathrm{M} 1$. The detection of an $(e, 2 e)$ ionization event, occurring shortly after ionization at time $t_{i}$, triggers the extraction of ions by raising the potentials of $\mathrm{M} 1$ and the needle to their optimum values for ion focusing. The electron beam and needle potentials are periodically pulsed to mitigate against the buildup of low-energy deuterons in the vicinity of the target region. See text for details.

Without the application of electron-beam pulsing and ion-cleaning cycles, a problem would have arisen from lowenergy deuterons, predominantly generated through GSD, accumulating in the neighborhood of the interaction volume between $(e, 2 e)$-instigated deuteron extractions. In that case, the deuteron associated with a measured $(e, 2 e)$ event would have been accompanied by many other deuterons created at earlier times whose $(e, 2 e)$ pairs were undetected. The presence of such a deuteron background would have greatly reduced the accuracy of the data. Furthermore, as we employed only single-hit time-to-digital converters in our time measurements, the measurement of multiple deuterons in a given extraction would have necessarily distorted the measured deuteron energy distribution as only the first detected deuteron in each extraction pulse is registered; thus, by employing the above pulsing scheme high levels of background deuterons were avoided. By implementing the timing technique illustrated in Fig. 4, we were able to achieve a triple-coincidence electron-electrondeuteron count rate of around 1 count/s for dissociative ionization with an associated deuteron background rate of $0.1 \mathrm{~Hz}$ at a time-averaged beam current of around $60 \mathrm{pA}$. In particular, the efficiency loss resulting from pulsing the electron beam with a $30 \%$ duty cycle was more than compensated for by the massive reduction in background signal. The small background that remains is mainly due to GSD-related $(e, 2 e)$ events, which dominate the $(e, 2 e)$ count rates. These are easily eliminated due to their much-lower associated electron binding energies. The remaining background events 
are spread over a large area of the two-dimensional phase space defined by deuteron kinetic energy and electron binding energy. To take an approximate account of the effects of the remaining background-deuteron contribution in the spectra we present here, we have subtracted the recorded $(e, 2 e)$ deuteron energy distribution for nondissociative ionization from that dissociative ionization with a scale factor chosen to achieve zero counts in regions of the dissociative-ionization energy spectrum where no states exist.

Since our previous measurement on $\mathrm{H}_{2}$ [24] and $\mathrm{N}_{2}$ [27], the electrical shielding of the detector circuits against highfrequency pickup from the few-nanosecond rise times of the extraction and needle potentials was greatly improved, as was the response time of our pulsing electronics. As a result, considerably higher extraction fields could be employed and the range of accessible fragment-ion energies could be extended. In addition, switching from the molecular target $\mathrm{H}_{2}$ to $\mathrm{D}_{2}$ reduced, by a factor of $\sqrt{2}$, the distance traversed by the ionic fragments between the instant of ionization $t_{i}$ and the time of extraction $t_{\text {ext }}$, improving ion focusing and further extending the range of ion energies which could be measured. Together these changes and improvements extended the energy range in which fragments could be collected and momentum analyzed over the full $4 \pi$ solid angle of emission to $12 \mathrm{eV}$. As a result we are able to measure transitions to the dissociative $2 p \sigma_{u}$ excited molecular-ion state, in addition to transitions to the $2 s \sigma_{g}$ and $2 p \pi_{u}$ excited states which we measured for $\mathrm{H}_{2}$. Transitions to the former state are associated with higher average deuteron energies than those to the latter two (see Fig. 2). The formula for appearance energy $A(n)$ [Eq. (2)] was employed to sort events according to whether they corresponded to transitions to the $2 p \sigma_{u}(n=1)$ or to the unresolved $2 s \sigma_{g}$ and $2 p \pi_{u}(n=2)$ states which possess a common dissociation limit, respectively. An appearance energy resolution of better than $2 \mathrm{eV}$ full width at half maximum (FWHM) was achieved for all momentum coordinates within the range of measured momentum phase space. Given this fact and the fact that $A(n=2)$ and $A(n=1)$ are separated by $10.2 \mathrm{eV}$, transitions to the $2 p \sigma_{u}$ and the $2 s \sigma_{g}$ and $2 p \pi_{u}$ states could be unambiguously distinguished from one another.

\section{FOUR-BODY DISTORTED-WAVE THEORY}

\section{A. Formalism}

In this section we present the theoretical framework within which calculations to describe the experimental data were performed. We emphasize here that an accurate description of the DEE dissociative-ionization process presents a great challenge, not only due to the inherent difficulty in describing electron scattering in a two-centered scattering potential, but also because the reaction involves a two-electron excitation leading to an electronic excitation of the residual molecular ion. Consequently, the DEE process poses a much greater theoretical challenge than that presented by the GSD process treated previously $[11,12]$ and one which must be addressed through a number of simplifying approximations to make the problem tractable.
The exact $T$ matrix for electron-impact excitationionization of oriented $\mathrm{D}_{2}$ can be written as

$$
T_{\mathrm{fi}}=\left\langle\Psi_{f}\left|H-H_{0}\right| \Phi_{i}\right\rangle
$$

where $H$ is the full Hamiltonian for the system, $H_{0}$ is an approximate initial-state Hamiltonian, and the wave functions $\Psi_{f}$ and $\Phi_{i}$ are eigenfunctions of the two Hamiltonians

$$
\begin{aligned}
& H\left|\Psi_{f}\right\rangle=E\left|\Psi_{f}\right\rangle, \\
& H_{0}\left|\Phi_{i}\right\rangle=E\left|\Phi_{i}\right\rangle .
\end{aligned}
$$

For electron-impact excitation-ionization of $D_{2}$, the full Hamiltonian is given by

$$
H=H_{\text {target }}+K_{i}+V_{i},
$$

where $H_{\text {target }}$ is the Hamiltonian for a neutral target with eigenfunctions $\Psi_{\text {target }}, K_{i}$ is the kinetic energy of the projectile electron $i$, and $V_{i}$ is the initial-state interaction between the projectile and target and given by the expression

$$
V_{i}=-\frac{1}{r_{N 1}}-\frac{1}{r_{N 2}}+\frac{1}{r_{i 1}}+\frac{1}{r_{i 2}} .
$$

Here $-\frac{1}{r_{N j}}$ is the interaction of the projectile electron with nucleus $j$, and $\frac{1}{r_{i j}}$ is the interaction of the projectile electron with electron $j$. In the distorted-wave approximation, the approximate initial-state Hamiltonian is given by

$$
H_{0}=H_{\text {target }}+K_{i}+U_{i},
$$

where $U_{i}$ is the an initial-state spherically symmetric approximation for the projectile target interaction $V_{i} . U_{i}$ is given by the expression

$$
U_{i}=U_{\mathrm{ele}}+U_{\mathrm{Nuc}},
$$

with $U_{\text {ele }}$ a spherically symmetric approximation for the interaction between the projectile electron and the target electrons, which is obtained from the quantum mechanical charge density of the target, and the nuclear contribution $U_{\text {Nuc }}$ is the interaction between the projectile electron and the two deuterons averaged over all orientations. Averaging the nuclei over all orientations is equivalent to putting the total nuclear charge of 2 on a thin spherical shell whose radius is the distance of the nuclei from the center of mass (c.m.). The eigenfunctions of the distorted-wave Hamiltonian (7) are given by

$$
\left|\Phi_{i}\right\rangle=\left|\Psi_{\text {target }}\left(\boldsymbol{r}_{\mathbf{1}}, \boldsymbol{r}_{\mathbf{2}}\right) \chi_{i}^{+}\left(\vec{k}_{i}, \boldsymbol{r}_{\mathbf{0}}\right)\right\rangle,
$$

where $\chi_{i}^{+}\left(\vec{k}_{i}, \boldsymbol{r}_{\mathbf{0}}\right)$ is a continuum-state distorted wave for wave number $\vec{k}_{i}$ and the + indicates outgoing wave boundary conditions. We initially tried using an accurate numerical wave function for $\Psi_{\text {target }}\left(\boldsymbol{r}_{1}, \boldsymbol{r}_{2}\right)$ but it quickly became clear that, even with a generous XSEDE grant, it was not practical to use this wave function. Consequently, we instead used the approximation

$$
\Psi_{\text {target }}\left(\boldsymbol{r}_{\mathbf{1}}, \boldsymbol{r}_{\mathbf{2}}\right) \approx \Psi_{\mathrm{Dy}}^{1 s}\left(\boldsymbol{r}_{\mathbf{1}}\right) \Psi_{\mathrm{Dy}}^{1 s}\left(\boldsymbol{r}_{\mathbf{2}}\right),
$$

where $\Psi_{\text {Dy }}^{1 s}$ is the ground-state Dyson wave function.

The exact wave functions for each final-state wave function are approximated as a product of wave functions for each of 
the final three particles and the final-state Coulomb interaction between the two continuum electrons

$$
\Psi_{f}\left(\boldsymbol{r}_{\mathbf{0}}, \boldsymbol{r}_{\mathbf{1}}, \boldsymbol{r}_{\mathbf{2}}\right) \approx \chi_{a}^{-}\left(\vec{k}_{a}, \boldsymbol{r}_{\mathbf{0}}\right) \chi_{b}^{-}\left(\vec{k}_{b}, \boldsymbol{r}_{\mathbf{1}}\right) \phi_{\mathrm{Ion}}\left(\boldsymbol{r}_{\mathbf{2}}\right) C_{\mathrm{se}}\left(\boldsymbol{r}_{\mathbf{0 1}}\right)
$$

Here $\chi_{a}^{-}\left(\vec{k}_{a}, \boldsymbol{r}_{\mathbf{0}}\right)$ is the final-state distorted-wave function for the scattered projectile with wave number $\left(\vec{k}_{a}\right), \chi_{b}^{-}\left(\vec{k}_{b}, \boldsymbol{r}_{1}\right)$ is the distorted wave for the ejected electron, $\phi_{\text {Ion }}\left(\boldsymbol{r}_{2}\right)$ is the Dyson wave function for the excited state of $\mathrm{D}_{2}{ }^{+}$, and $C_{\text {se }}\left(\boldsymbol{r}_{\mathbf{0 1}}\right)$ and is the Coulomb interaction between the scattered projectile and the ejected electron, which is normally called the postcollision interaction (PCI). The final-state distorted waves are calculated similarly to the initial-state distorted waves except that the spherically symmetric potential for the final ion is used. Consequently, the M4DW $T$ matrix is given by

$$
\begin{aligned}
T_{\mathrm{fi}}= & \left\langle\chi_{a}^{-}\left(\vec{k}_{a}, \boldsymbol{r}_{\mathbf{0}}\right) \chi_{b}^{-}\left(\vec{k}_{b}, \boldsymbol{r}_{\mathbf{1}}\right) \phi_{\mathrm{Ion}}\left(\boldsymbol{r}_{\mathbf{2}}\right) C_{\mathrm{se}}\left(\boldsymbol{r}_{\mathbf{0 1}}\right)\right. \\
& \left.\times\left|V_{i}-U_{i}\right| \Psi_{\mathrm{Dy}}^{1 s}\left(\boldsymbol{r}_{\mathbf{1}}\right) \Psi_{\mathrm{Dy}}^{1 s}\left(\boldsymbol{r}_{\mathbf{2}}\right) \chi_{i}^{+}\left(\vec{k}_{i}, \boldsymbol{r}_{\mathbf{0}}\right)\right\rangle .
\end{aligned}
$$

Since there are three active particles in this $T$ matrix, the evaluation requires a full nine-dimensional integration which we perform numerically [32,33]. In our formalism, alignment-dependent effects emerge through the dependence of the excitation probabilities on the overlap of the initial-state Dyson wave functions $\Psi_{\text {Dy }}^{1 s}$ with the final-state Dyson ion wave function $\phi_{\text {Ion }}\left(\boldsymbol{r}_{2}\right)$.

\section{B. Normalization of experiment to theory}

The triple differential cross section (TDCS), which is compared to the experimental results of this paper, is related to the $T$ matrix $T_{\mathrm{fi}}$ through the expression:

$$
\begin{aligned}
\operatorname{TDCS}\left(\theta_{a}, \phi_{a}, \theta_{b}, \phi_{b}, E_{b}\right) & =\frac{d \sigma^{5}}{d \Omega_{a} d \Omega_{b} d E_{b}} \\
& =\frac{1}{(2 \pi)^{5}} \frac{k_{a} k_{b}}{k_{i}}\left|T_{\mathrm{fi}}\right|^{2} .
\end{aligned}
$$

We measure over a $10^{\circ}$ to $50^{\circ}$ interval for $\theta_{a}$ (average azimuthal angle $\bar{\phi}_{a}=0^{\circ}$ ) and a $40^{\circ}$ to $80^{\circ}$ interval for $\theta_{b}$ (average angle $\bar{\phi}_{b}=180^{\circ}$ ), accepting electrons through a constant-width circular entrance aperture which is centered on the interaction region. The range $\Delta \phi_{a}$ of $\phi_{a}$ values and the range $\Delta \phi_{b}$ of $\phi_{b}$ values for measured emitted electrons varies with the angles $\theta_{a}$ and $\theta_{b}$, respectively. At $\theta_{a(b)}=90^{\circ}$ (outside the capture range of both analyzers), the range of $\phi_{a(b)}$, subtended at the interaction region, is bounded by the values $\pm \Gamma^{\circ}$, where $\Gamma \cong 2^{\circ}$ in our experiment. To a good approximation, within the two polar angular acceptance ranges of the electron analyzers, values for $\phi_{a(b)}$ are bounded by the limits $\Gamma_{a(b)}$, where $\Gamma_{a(b)}=\Gamma / \sin \theta_{a(b)}$. Thus, $\Delta \phi_{a(b)}$ can be approximated by the expression $\Delta \phi_{a(b)}=2 \Gamma / \sin \theta_{a(b)}$.

To relate calculated TDCS values to our measured $(e, 2 e)$ event rates we must average them over the range of $\theta$ and $\phi$ values contributing to each experimental data point; we denote the resultant quantity as TDCS $_{\mathrm{av}}$. The integration is performed over the range of polar $\left(\Delta \theta_{a}, \Delta \theta_{b}\right)$ and azimuthal $\left(\Delta \phi_{a}, \Delta \phi_{b}\right)$ angles over which counts are summed in the analysis of the experimental data. In general we have

$$
\mathrm{TDCS}_{\mathrm{av}}=\int_{\Delta \theta_{a}} \int_{\Delta \theta_{b}} \int_{\Delta \phi_{a}} \int_{\Delta \phi_{b}} \frac{d \sigma^{5}\left(\theta_{a}, \phi_{a}, \theta_{b}, \phi_{b}, E_{b}\right)}{d \Omega_{a} d \Omega_{b} d E_{b}} d \phi_{a} d \phi_{b} d \theta_{a} \sin \theta_{a} d \theta_{b} \sin \theta_{b} / \int_{\Delta \Omega_{a}} d \Omega_{a} \int_{\Delta \Omega_{b}} d \Omega_{b},
$$

where $\Delta \Omega_{a(b)}$ represents the acceptance solid angles for the electron analyzers accepting the fast $a$ and slow $b$ scattered electrons, respectively. For the present experimental arrangement, as the ranges of $\Delta \phi_{a}$ and $\Delta \phi_{b}$ are small, we can approximate the integrand by its value at the coordinate $\left(\phi_{a}=0^{\circ}, \phi_{b}=180^{\circ}\right)$, namely $d \sigma^{5}\left(\theta_{a}, \phi_{a}=0^{\circ}, \theta_{b}, \phi_{b}=180^{\circ}, E_{b}\right) / d \Omega_{a} d \Omega_{b} d E_{b}$. Substituting for $\Delta \phi_{a(b)}$ and through rearrangement we obtain

$$
\begin{aligned}
\mathrm{TDCS}_{\mathrm{av}} & \cong \int_{\Delta \theta_{a}} \int_{\Delta \theta_{b}} \frac{d \sigma^{5}\left(\theta_{a}, \phi_{a}=0^{\circ}, \theta_{b}, \phi_{b}=180^{\circ}, E_{b}\right)}{d \Omega_{a} d \Omega_{b} d E_{b}} d \theta_{a} \sin \theta_{a} d \theta_{b} \sin \theta_{b} \int_{\Delta \phi_{a}} d \phi_{a} \int_{\Delta \phi_{b}} d \phi_{b} / \int_{\Delta \Omega_{a}} d \Omega_{a} \int_{\Delta \Omega_{b}} d \Omega_{b} \\
& =\frac{1}{\Delta \theta_{a} \Delta \theta_{b}} \int_{\Delta \theta_{a}} \int_{\Delta \theta_{b}} \frac{d \sigma^{5}\left(\theta_{a}, \phi_{a}=0^{\circ}, \theta_{b}, \phi_{b}=180^{\circ}, E_{b}\right)}{d \Omega_{a} d \Omega_{b} d E_{b}} d \theta_{a} d \theta_{b} .
\end{aligned}
$$

To reduce statistical fluctuations between experimental data points, $(e, 2 e)$ events were summed over the range of polar angles $\Delta \theta_{b}$, where $40^{\circ} \leqslant \theta_{b} \leqslant 80^{\circ}$ and over the $10^{\circ}$ range of azimuthal angles $\Delta \theta_{a}\left(j^{\circ}\right)$, where $j^{\circ}=15^{\circ}+(i-1) \times 10^{\circ}, i=1-4$, and $i \times 10^{\circ} \leqslant \theta_{a} \leqslant(i+1) \times 10^{\circ}$. The resultant experimental counts (presented in Figs. 5 and 6 of this paper) are then ascribed to the mean angular values $j^{\circ}$ of the respective angular ranges $\Delta \theta_{a}\left(j^{\circ}\right)$. To facilitate the comparison of theory with experiment and to reduce computational overheads we make a further approximation in Eq. (15); we replace the integrand by its value at the angular coordinates $\theta_{a}=j^{\circ}$ :

$$
\begin{aligned}
\operatorname{TDCS}_{\mathrm{av}}\left[\theta_{a}=j^{\circ}\right] & \cong \frac{1}{\Delta \theta_{a} \Delta \theta_{b}} \times \int_{40^{\circ}}^{80^{\circ}} \frac{d \sigma^{5}\left(\theta_{a}=j^{\circ}, \phi_{a}=0^{\circ}, \theta_{b}, \phi_{b}=180^{\circ}, E_{b}\right)}{d \Omega_{a} d \Omega_{b} d E_{b}} d \theta_{b} \int_{\Delta \theta_{a}} d \theta_{a} \\
& =\frac{1}{\Delta \theta_{b}} \int_{40^{\circ}}^{80^{\circ}} \frac{d \sigma^{5}\left(\theta_{a}=j^{\circ}, \phi_{a}=0^{\circ}, \theta_{b}, \phi_{b}=180^{\circ}, E_{b}\right)}{d \Omega_{a} d \Omega_{b} d E_{b}} d \theta_{b},
\end{aligned}
$$

where $\Delta \theta_{b}=40^{\circ}$. 


\section{RESULTS}

In Figs. 5(a) to 5(d) we present triple-coincidence count rates for transitions to the unresolved $2 s \sigma_{g}$ and $2 p \pi_{u}$ and to the $2 p \sigma_{u}$ excited states and for selected molecular alignments of the $\mathrm{D}_{2}{ }^{+}$ion as a function of the scattering angle $\theta_{a}$ for $100-\mathrm{eV}$ scattered electrons. For each alignment direction the data comprises events for which deuterons are emitted within a cone of $\pm 15^{\circ}$, corresponding to $3.3 \%$ of the spherical surface. As all transitions and all alignments were measured simultaneously under identical experimental conditions, their relative strengths are reflected in the respective coincidence count rate scales of the four panels. As mentioned earlier, deuterons emitted over the full $4 \pi$ solid angle are detected. This enables the dependence of the dissociative-ionization rate on all deuterium alignment-directions to be explored. To aid interpretation of the underlying physics, however, ionization rates are presented for five specific high-symmetry alignment directions of the deuterium internuclear axis with respect to both the momentum direction of the incident electron and the direction of the momentum transfer $\boldsymbol{K}$. The alignment directions denoted as $\mathrm{D}_{X}, \mathrm{D}_{Y}$, and $\mathrm{D}_{Z}$ correspond, respectively, to deuterium molecules oriented perpendicular to the primary beam direction and within the $x-z$ scattering plane $\left(\mathrm{D}_{X}\right.$ alignment), perpendicular to the primary beam and perpendicular to the scattering plane ( $\mathrm{D}_{Y}$ alignment) and molecules oriented along the primary beam direction ( $\mathrm{D}_{Z}$ alignment). Each lie parallel to one of the Cartesian coordinate axes $x, y$, and $z$ (see Fig. 1). Two further alignment directions within the $x-z$ scattering plane are defined. One $\left(\mathrm{D}_{K}\right.$ alignment) describes an alignment along the direction of momentum transfer $\boldsymbol{K}$ and the other $\left(\mathrm{D}_{\boldsymbol{K} \perp}\right.$ alignment) defines an alignment perpendicular to $\boldsymbol{K}$.

The triple-coincidence count rate is presented as a function of the scattering angle $\theta_{a}$ of the $100-\mathrm{eV}$ scattered electron. Varying $\theta_{a}$ is equivalent to varying the momentum transfer both in magnitude and direction. For example, for transitions to the $2 p \sigma_{u}$ state, varying the value of $\theta_{a}$ from $15^{\circ}$ to $45^{\circ}$ varies the magnitude of momentum transfer $|\boldsymbol{K}|$ from 1.2 to 2.6 a.u. and the direction $\theta_{\boldsymbol{K}}$ of momentum transfer from $37^{\circ}$ to $50^{\circ}$. Furthermore, as only around $5 \%$ of all measured $(e, 2 e)$ ionization events are accompanied by fragmentation of the residual $\mathrm{D}_{2}{ }^{+}$ion, the data are averaged over the slow-electron scattering angle $\theta_{b}$ and over the electron-energy pass bands $\Delta E_{a}$ and $\Delta E_{b}$ to reduce the statistical spread of the presented data. In spite of this integration, dramatic alignment-dependent effects remain.

Figure 5(a) shows the measured electron-electron-ion triple-coincidence rate for transitions to the $2 s \sigma_{g}$ and $2 p \pi_{u}$ excited states of the $\mathrm{D}_{2}{ }^{+}$ion as a function of the scattering angle $\theta_{a}$ of the $100-\mathrm{eV}$ scattered electron for the $\mathrm{D}_{X}, \mathrm{D}_{Y}$, and $\mathrm{D}_{Z}$ molecular alignments. As the fragment-deuteron energy
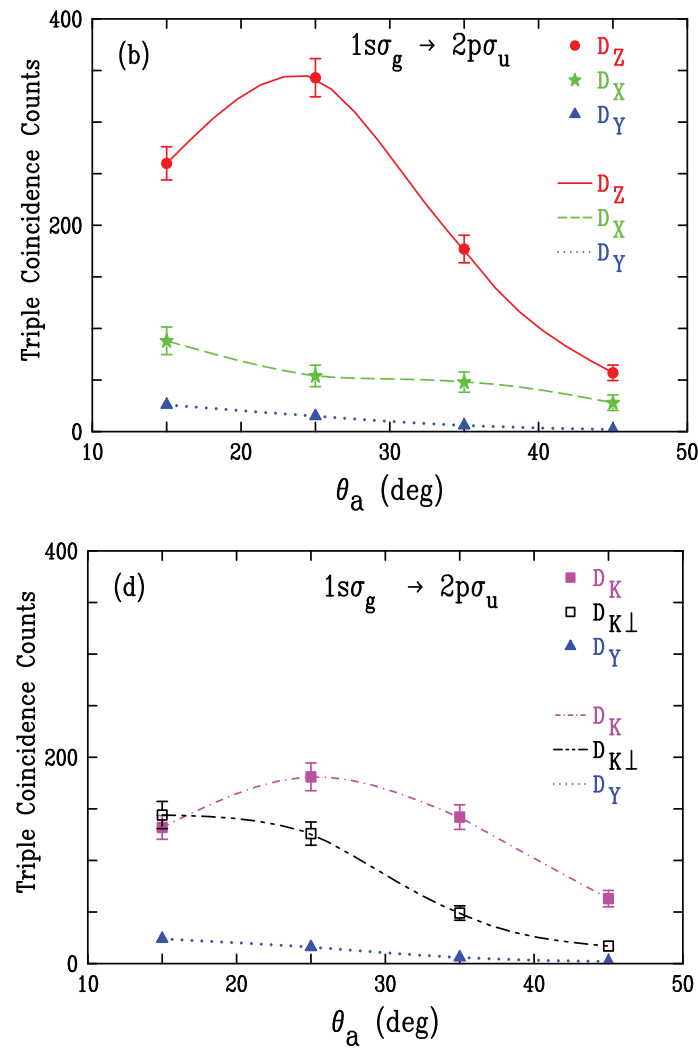

FIG. 5. (Color online) Triple-coincidence counts for transitions to the $2 s \sigma_{g}$ and $2 p \pi_{u}$ [panels (a) and (c)] and $2 p \sigma_{u}$ states [panels (b) and (d)] of $\mathrm{D}_{2}{ }^{+}$as a function of the fast-electron scattering-angle $\theta_{a}$. Panels (a) and (b) show experimental results for the three molecular alignments $\mathrm{D}_{X}, \mathrm{D}_{Y}$, and $\mathrm{D}_{Z}$. Panels (c) and (d) show experimental results for the three molecular alignments $\mathrm{D}_{K}, \mathrm{D}_{K \perp}$, and $\mathrm{D}_{Y}$. The data have been averaged over the slow-electron scattering angle $\theta_{b}$ and have been fitted with second-order polynomials to aid visualization of the trends. As all results were accumulated simultaneously under identical experimental conditions, the relative count rates between data in all four panels therefore reflect the relative strengths of their associated cross sections. 
distributions associated with transitions to the individual $2 s \sigma_{g}$ and $2 p \pi_{u}$ states strongly overlap in energy (see [26]), their individual contributions cannot be resolved. To assist the eye by highlighting the dependency of count rate on the alignment and momentum transfer, the data have been fitted with second order polynomials. Immediately evident is the strong alignment dependence of the dissociative-ionization rate as reflected by the alignment dependence of the coincidence count rates. Of the three alignment directions considered, side-on collisions of the projectile with the deuterium molecule ejected out of the scattering plane $\left(\mathrm{D}_{Y}\right.$ alignment) leads to the highest rates of dissociative ionization. Side-on collisions of the projectile with the deuterium molecule and with the deuteron ejected in the scattering plane $\left(D_{X}\right.$ alignment) exhibits a smaller rate, and "end-on" collisions $\left(\mathrm{D}_{Z}\right.$ alignment) with the molecule giving rise to smallest rate overall. Given that the present measurements were performed under identical kinematical conditions to our previous study of $\mathrm{H}_{2}$ and given that the electronic structure (as opposed to the vibrational structure) of deuterium and hydrogen molecules is essentially the same, one would expect that the present results and those published by us previously for $\mathrm{H}_{2}$ (Fig. 5 of [24]) would be the same. This is indeed the case when one compares the relative transition strengths for the $\mathrm{D}_{Y}$ and $\mathrm{D}_{Z}$ alignments. However, when one compares the relative count rates for all three alignments, $\mathrm{D}_{X}$ (labeled $P_{X}$ in [24]) is different. Subsequent detailed checking revealed an error in our analysis for the $\mathrm{P}_{X}$ alignment data of $\mathrm{H}_{2}$, accounting for this discrepancy.
Figure 5(b) shows analogous results for transitions to the $2 p \sigma_{u}$ dissociative state. Comparing to the results in Fig. 5(a), a dramatic transition dependence on the rate of dissociative ionization is seen. In Fig. 5(b) end-on collisions, which were the least-favored molecular alignment to lead to dissociative ionization in Fig. 5(a), now dominates for transitions to the $2 p \sigma_{u}$ state. Of the side-on collisions ( $\mathrm{D}_{X}$ and $\mathrm{D}_{Y}$ alignments), deuteron emission in the scattering plane $\left(\mathrm{D}_{X}\right.$ alignment) is strongly favored relative to emission out of the scattering plane ( $\mathrm{D}_{Y}$ alignment). The $\mathrm{D}_{Y}$ alignment now has the lowest cross section, whereas for the $2 s \sigma_{g}$ and $2 p \pi_{u}$ transitions it possessed the largest [Fig. (5a)].

Figures 5(c) and 5(d) show the same $\mathrm{D}_{Y}$ alignment data as in 5(a) and 5(b), but additionally data for the $D_{K}$ and $\mathrm{D}_{K \perp}$ alignments. These particular alignment directions, which depend on $\theta_{a}$, are chosen to facilitate a comparison of the present results with findings from photoionization studies, where dipole selection rules can account for strong alignment dependence in the angular distributions of photoelectrons. However, the present results occupy a kinematic regime well removed from the optical limit of high electron-impact energies and negligible values for momentum transfer $\boldsymbol{K}$. Thus, while one might anticipate, a priori, that some physical insight into the present observations might be obtained from considering dipole selection rules, a fully quantum mechanical treatment is required for an accurate interpretation of the data.

Figures 6(a) to 6(d) show the same experimental results in Figs. 5(a) to 5(d) but this time compared to the M4DW theory.
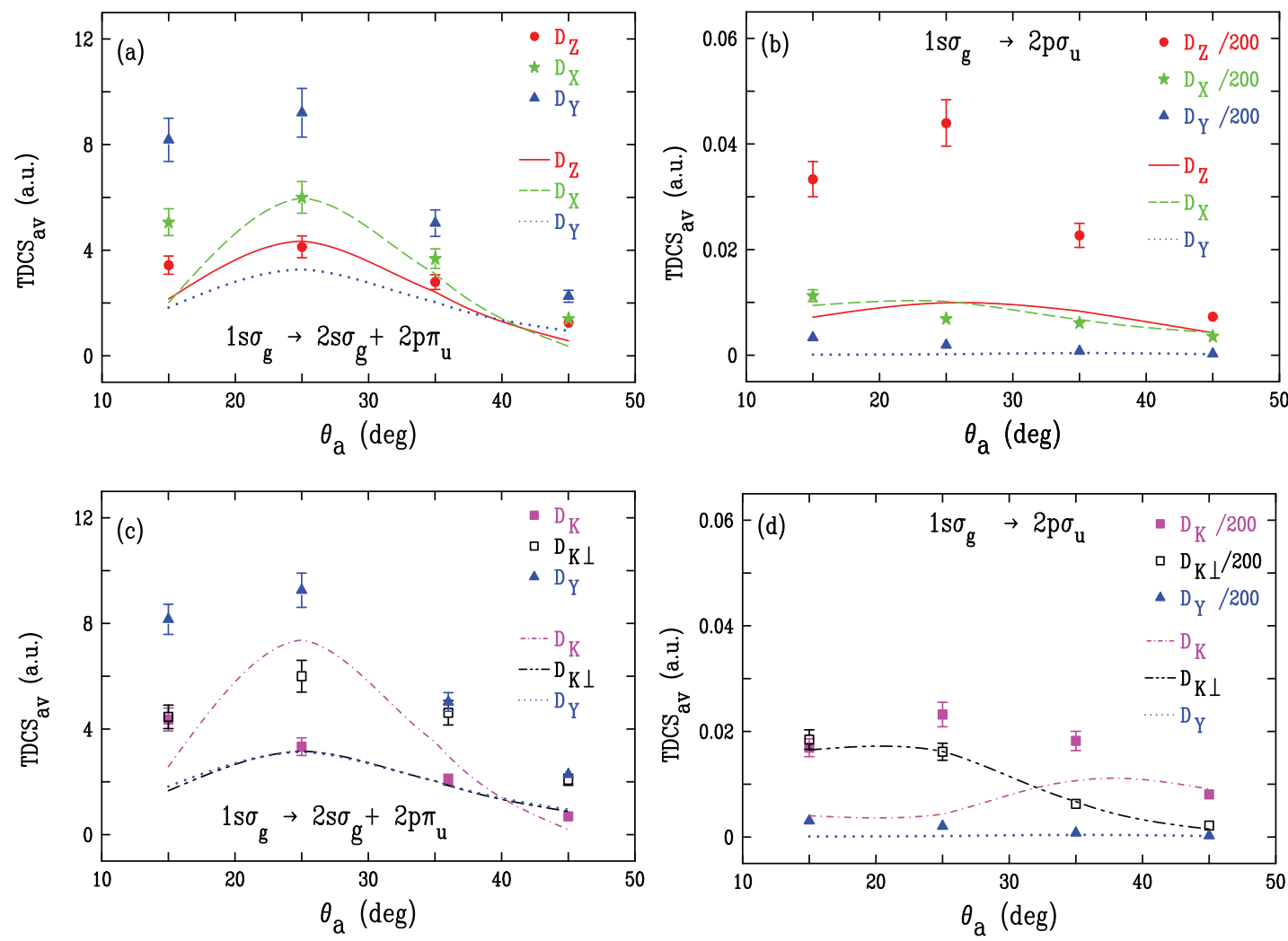

FIG. 6. (Color online) Same experimental data shown in Fig. 5 compared to M4DW calculations. The experimental results have been normalized to the calculations [averaged according to Eq. (16)] at the scattering angle $\theta_{a}=25^{\circ}$ for the $\mathrm{D}_{X}$ molecular-frame alignment of the $2 s \sigma_{g}$ and $2 p \pi_{u}$ transitions. 

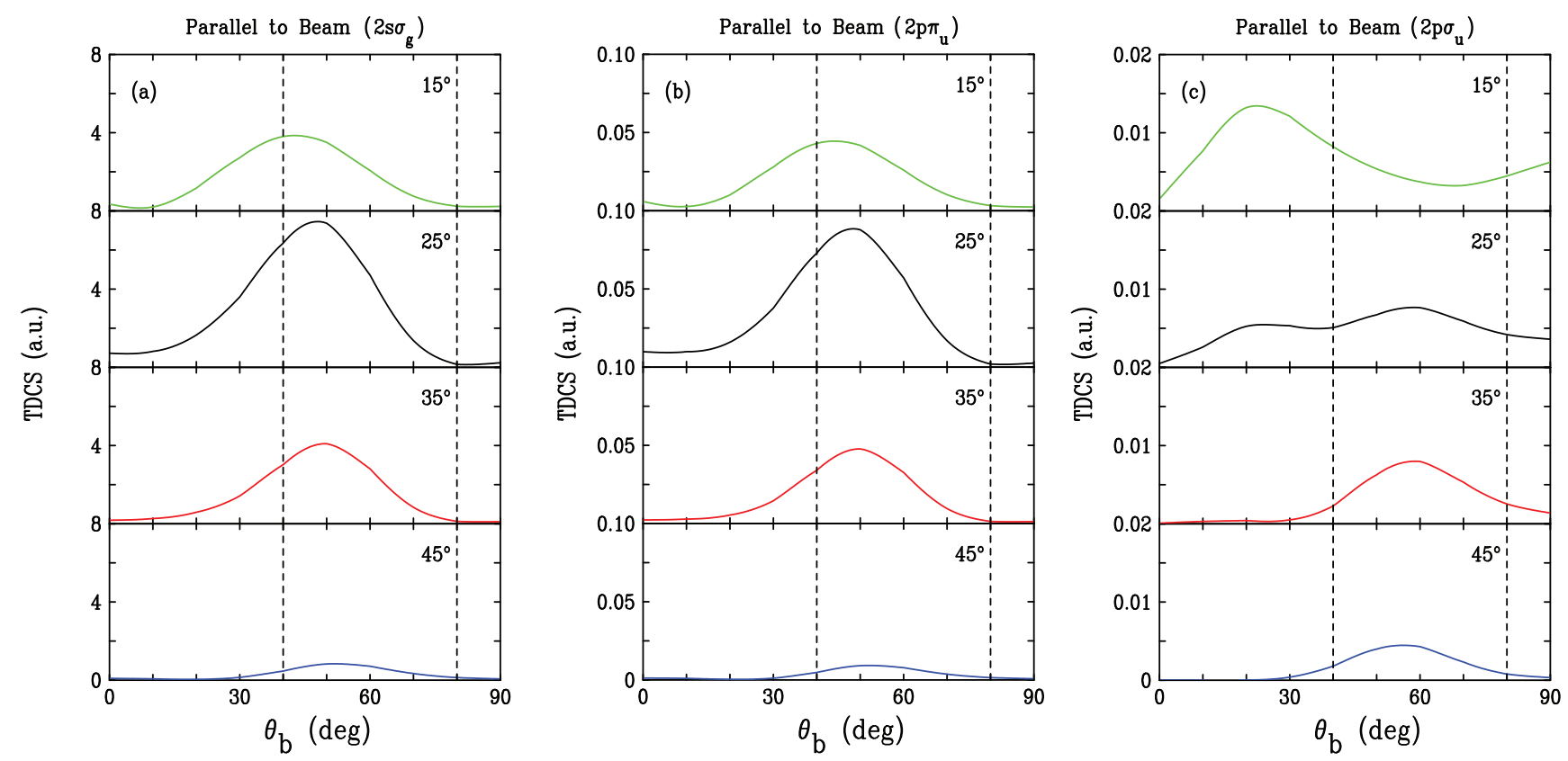

FIG. 7. (Color online) M4DW theoretical results for the three different excited states as a function of the ejected-electron scattering angle and for the $\mathrm{D}_{Z}$ alignment of the internuclear axis. The angles noted in each panel are the faster (projectile) electron scattering angles. To compare with experiment, the theoretical cross sections were integrated between the two vertical lines.

Normalization of the experimental results to the calculations was achieved at the scattering angle $\theta_{a}=25^{\circ}$ for the $2 s \sigma_{g}$ and $2 p \pi_{u}$ transitions and for the $\mathrm{D}_{X}$ molecular-frame alignment [Fig. 5(a)]. Figure 6(a) shows results for transitions to the $2 s \sigma_{g}$ and $2 p \pi_{u}$ excited states. The M4DW predicts the same order and relative magnitude as experiment for alignment in the scattering plane. However, for the alignment out of the scattering plane $\mathrm{D}_{Y}$, theory predicts this to be the weakest transition while in contrast experiment finds it to be the strongest. In Fig. 6(c) alignments along the $\mathrm{D}_{Y}, \mathrm{D}_{K}$, and $\mathrm{D}_{K} \perp$ directions are considered. In this case, experiment finds $\mathrm{D}_{K}$ has a similar magnitude to $\mathrm{D}_{Z}$ and $\mathrm{D}_{K \perp}$ has a similar magnitude to $\mathrm{D}_{X}$, with the $\mathrm{D}_{K} \perp$ alignment preferred over the $\mathrm{D}_{K}$. In contrast, theory predicts that the largest cross section should be found for the momentum-transfer direction (as was found in both experiment and theory for direct ionization of the ground state). Furthermore, it predicts almost identical results for the two directions perpendicular to momentum transfer, both in and out of the scattering plane, in contrast to the experimental findings.

For the isolated $2 p \sigma_{u}$ transition shown in Figs. 6(b) and $6(\mathrm{~d})$ and using the same normalization as in panel (a) (i.e., at the scattering angle $\theta_{a}=25^{\circ}$ for the combined $2 s \sigma_{g}$ and $2 p \pi_{u}$ transitions and for the $\mathrm{D}_{X}$ molecular-frame alignment) experiment finds a relative cross-section value that is 200 times larger than predicted by theory. Furthermore, experiment shows a much stronger dependence on alignment than theory, although the relative order for the predicted alignment dependencies is the same for both at larger values for $\theta_{a}$. While experiment finds a very small cross section for $\mathrm{D}_{Y}$, the theoretical cross section is zero to within numerical error due to the symmetry of the state. The small nonzero value found experimentally is most likely due to the summation of the data over the finite angular cone of $\pm 15^{\circ}$. Interestingly, both the shape and relative magnitude of the theoretical $\mathrm{D}_{X}, \mathrm{D}_{K \perp}$, and $\mathrm{D}_{Y}$ results agree with the experimental measurements. As a result, theory and experiment are in fairly good agreement for the shape and relative magnitudes for the $2 p \sigma_{u}$ state except for the beam $\left(D_{Z}\right)$ and momentum-transfer $\left(D_{K}\right)$ directions and the magnitudes relative to the $2 s \sigma_{g}$ and $2 p \pi_{u}$ states. We have previously found a similar result for electron-impact excitation-ionization of helium where the 4DW results were badly incorrect for the absolute magnitude of the cross section while giving reasonable agreement with the shape of the data [34].

As mentioned above, the experimental results were integrated over an acceptance angle between $40^{\circ}$ and $80^{\circ}$ for $\theta_{b}$ to improve statistics. Figure 7 shows the theoretical results for $\theta_{b}$ between zero and $90^{\circ}$ with vertical lines at $40^{\circ}$ and $80^{\circ}$. The theoretical results were integrated between the two vertical lines. This range was picked because it was expected that the cross sections would be largest in this angular range, which is the case for the larger scattered-projectile angles. However, for the smaller values of $\theta_{a}$, a significant part of the cross section lies outside the angular range. Also note the relative magnitudes of the cross sections for the three states. The scale for the $2 p \pi_{u}$ is 40 times smaller than the $2 s \sigma_{g}$, which means that the $2 s \sigma_{g}$ and $2 p \pi_{u}$ results are essentially all $2 s \sigma_{g}$. Also the $2 p \sigma_{u}$ scale is a factor of 400 times smaller than that for the $2 s \sigma_{g}$ state.

\section{CONCLUSION}

We have presented experimental and M4DW theoretical results for excitation-ionization of molecular $\mathrm{D}_{2}$. The alignment of the $D_{2}$ molecules was inferred by determining the momenta of emitted deuterons for three different excited states of $\mathrm{D}_{2}{ }^{+}$which dissociate immediately following ionization. 
A significant dependence of the dissociative-ionization cross section on both the molecular alignment and the symmetry of the excited $\mathrm{D}_{2}{ }^{+}$dissociative states was found in the results of both experiment and calculations. Discrepancies between the two data sets are, however, observed.

For the $2 s \sigma_{g}$ and $2 p \pi_{u}$ states, experiment found the largest cross section for a molecular alignment perpendicular to the scattering plane while theory predicted the largest cross sections for the alignment parallel to the momentum-transfer direction. Theory predicted the smallest cross sections for alignment perpendicular to the scattering plane. There was fairly good agreement between experiment and theory for the alignment directions parallel and perpendicular to the incident-beam direction. For the $2 p \sigma_{u}$ state, the relative magnitude of the experimental data is a factor of 200 larger than the theoretical prediction. However, there was otherwise reasonable agreement between experiment and theory with respect to the relative magnitude and shape of the cross sections for the different alignment directions. The only significant disagreements were for molecular alignments parallel to the electron-beam axis and parallel to the momentum-transfer direction. For the case of alignment perpendicular to the scattering plane, theory predicts a cross section of zero due to the symmetry of the state. This is supported by the measurements.

Although there are some encouraging aspects of the agreement between experiment and theory, there are also significant disagreements. In particular, the theory predicts very small values for the ratio of cross sections for transitions leading to ungerade relative to those for gerade states, some 200 times smaller than determined by experiment. Given the clean separation of measured events by appearance energy, and the very low background at these appearance energies, the authors expect the accuracy of the experimentally determined ratio to be dominated by the statistical error. In addition, theory predicts that the cross sections describing transitions to the $2 p \pi_{u}$ state are 40 times smaller than those for transitions to the $2 s \sigma_{g}$ states. The remaining disparities between theory and experiment are probably largely due to the rather crude wave function used for the initial state of the target. To check the importance of this wave function, we will repeat the calculation using a better configuration interaction wave function. However, there are approximations in the theory other than the elementary ground-state wave function that could be important, such as using continuum wave functions that are calculated using a scattering potential which has been averaged over all molecular orientations. Although this might logically seem to be more important than the ground-state wave-function approximation, we have previously found that the M4DW gives good agreement with experiment for the case of ionization of aligned $\mathrm{H}_{2}$ with the ion being left in the ground state $[9,10]$, so we assume that this would also be a good approximation if the ion is left in an excited state.

\section{ACKNOWLEDGMENTS}

E.A. and D.M. acknowledge support of the US National Science Foundation under Grant No. PHY-1068237 and XSEDE resources [35] provided by the Texas Advanced Computing Center (Grant No. TG-MCA07S029). XSEDE systems are hosted by Indiana University, LONI, NCAR, NCSA, NICS, ORNL, PSC, Purdue University, SDSC, TACC, and UC/ANL. C.G.N. would like to acknowledge the support of the National Natural Science Foundation of China under Contract No. 10704046. J.L., S.B., and E.W. thank Colin Dedman, Stephen Battisson, and Ross Tranter for their outstanding technical support at the Australian National University.
[1] V. I. Shematovich, R. E. Johnson, M. Michael, and J. G. Luhmann, J. Geophys. Res. 108, 5087 (2003).

[2] J. Liu, F. Sun, and H. Yu, Current Appl. Phys. 5, 625 (2005).

[3] I. IPolyi, P. Cicman, S. Denifl, V. Matejčík, P. Mach, J. Urban, P. Scheier, T. D. Märk, and Š. Matejčík, J. Mass Spectrom. 252, 228 (2006).

[4] J. Lower, M. Yamazaki, and M. Takahashi, in Fragmentation Processes, edited by Colm T. Whelan (Cambridge University Press, Cambridge, UK, 2013), p. 137.

[5] M. Takahashi, N. Watanabe, Y. Khajuria, K. Nakayama, Y. Udagawa, and J. H. D. Eland, J. Electron Spectrosc. Relat. Phenom. 141, 83 (2004).

[6] M. Takahashi, N. Watanabe, Y. Khajuria, Y. Udagawa, and J. H. D. Eland, Phys. Rev. Lett. 94, 213202 (2005).

[7] M. Takahashi and Y. Udagawa, in Nanoscale Interactions and Their Applications: Essays in Honour of Ian McCarthy, edited by F. Wang and M. J. Brunger (Transworld Research Network, Kerala, India, 2007), p. 157.

[8] M. Takahashi, Bull. Chem. Soc. Jpn. 82, 751 (2009).

[9] A. Senftleben, T. Pflüger, X. Ren, O. Al-Hagan, B. Najjari, D. Madison, A. Dorn, and J. Ullrich, J. Phys. B 43, 081002 (2010).

[10] A. Senftleben, O. Al-Hagan, T. Pflüger, X. Ren, D. Madison, A. Dorn, and J. Ullrich, J. Chem. Phys. 133, 044302 (2010).
[11] X. Ren, T. Pflüger, S. Xu, J. Colgan, M. S. Pindzola, A. Senftleben, J. Ullrich, and A. Dorn, Phys. Rev. Lett. 109, 123202 (2012).

[12] X. Ren, A. Senftleben, T. Pflüger, A. Dorn, J. Colgan, M. S. Pindzola, O. Al-Hagan, D. H. Madison, I. Bray, D. V. Fursa, and J. Ullrich, Phys. Rev. A 82, 032712 (2010).

[13] J. Gao, J. L. Peacher, and D. H. Madison, J. Chem. Phys. 123, 204302 (2005).

[14] J. Gao, D. H. Madison, and J. L. Peacher, J. Chem. Phys. 123, 204314 (2005).

[15] J. Gao, D. H. Madison, and J. L. Peacher, J. Phys. B 39, 1275 (2006).

[16] C. R. Stia, O. A. Fojon, P. F. Weck, J. Hanssen, and R. D. Riverola, J. Phys. B 36, L257 (2003).

[17] O. Al-Hagan, C. Kaiser, D. Madison, and A. J. Murray, Nat. Phys. 5, 59 (2009).

[18] M. S. Pindzola, F. Robicheaux, S. D. Loch, J. C. Berengut, T. Topcu, J. Colgan, M. Foster, D. C. Griffin, C. P. Balance, D. R. Schultz, T. Minami, N. R. Badnell, M. C. Witthoeft, D. R. Plante, D. M. Mitnik, J. A. Ludlow, and U. Kleiman, J. Phys. B 40, R39 (2007).

[19] J. Colgan, M. S. Pindzola, F. Robicheaux, C. Kaiser, A. J. Murray, and D. H. Madison, Phys. Rev. Lett. 101, 233201 (2008). 
[20] J. Colgan, O. Al-Hagan, D. H. Madison, C. Kaiser, A. J. Murray, and M. S. Pindzola, Phys. Rev. A 79, 052704 (2009).

[21] J. Colgan and M. S. Pindzola, J. Phys.: Conf. Ser. 288, 012001 (2011).

[22] I. Bray and D. V. Fursa, Phys. Rev. A 54, 2991 (1996).

[23] A. T. Stelbovics, I. Bray, D. V. Fursa, and K. Bartschat, Phys. Rev. A 71, 052716 (2005).

[24] S. Bellm, J. Lower, E. Weigold, and D. W. Mueller, Phys. Rev. Lett. 104, 023202 (2010).

[25] R. N. Zare, Mol. Photochem. 4, 1 (1972).

[26] T. E. Sharp, At. Data 2, 119 (1970).

[27] S. Bellm, J. Lower, D. Mueller, and E. Weigold, J. Phys. Conf. Ser. 212, 012005 (2010).

[28] J. Lower, R. Panajotovic, S. Bellm, and E. Weigold, Rev. Sci. Instrum. 78, 111301 (2007).
[29] O. Jagutzki, V. Mergel, K. Ullmann-Pfleger, L. Spielberger, U. Spillmann, R. Dörner, and H. Schmidt-Böcking, Nucl. Instrum. Methods Phys. Res., Sect. A 477, 244 (2002).

[30] J. Ullrich, R. Moshammer, A. Dorn, R. Dörner, L. P. H. Schmidt, and H. Schmidt-Böcking, Rep. Prog. Phys. 66, 1463 (2003).

[31] SIMION, ion source software, P.O. Box 2726, Idaho Falls, ID 83404, USA.

[32] A. L. Harris, M. Foster, J. L. Peacher, and D. H. Madison, J. Phys. B 41, 135203 (2008).

[33] A. L. Harris, J. L. Peacher, D. H. Madison, and J. Colgan, Phys. Rev. A 80, 062707 (2009).

[34] S. Bellm, J. Lower, E. Weigold, I. Bray, D. V. Fursa, K. Bartschat, A. L. Harris, and D. H. Madison, Phys. Rev. A 78, 032710 (2008).

[35] C. Catlett et al., in HPC and Grids in Action, edited by Luco Grandinetti (IOS Press, Amsterdam, 2007). 